\title{
Non-contrasted Computed Tomography for the Accurate Measurement of Liver Steatosis in Obese Patients
}

\author{
Nathan J. Shores · Kerry Link • Adolfo Fernandez • \\ Kim R. Geisinger $\cdot$ Matt Davis $\cdot$ Tam Nguyen · \\ Janet Sawyer $\cdot$ Larry Rudel
}

Received: 24 November 2010/ Accepted: 27 January 2011/Published online: 12 February 2011

(C) The Author(s) 2011. This article is published with open access at Springerlink.com

\begin{abstract}
Background Hepatic macrosteatosis (HMS) is prevalent among high BMI patients, but a lack of validation of noninvasive measures of liver fat hampers non-alcoholic liver disease (NAFLD) investigation in general. Recent work suggests BMI adjusted, non-contrasted computed tomography (nc-CT) attenuation data (Hounsfield units) reflects liver fat accumulation in a normal weight population. However, this and other CT-based HMS studies have only approximated macrosteatosis (\%) histologically, but have not validated findings with chemical liver triglyceride (TG) concentrations (mg/gram protein). Also, all previous CT based steatosis studies excluded high BMI subjects, whose
\end{abstract}

\section{N. J. Shores $(\square)$}

Division of Gastroenterology and Transplant Hepatology, University of California, San Francisco, 505 Parnassus Street,

San Francisco, CA 94143, USA

e-mail: Nathan.Shores@UCSF.edu

\section{K. Link}

Center for Biomedical Imaging, Wake Forest University Health

Sciences, Winston-Salem, NC, USA

\section{A. Fernandez}

Department of Surgery, Wake Forest University Health

Sciences, Winston-Salem, NC, USA

K. R. Geisinger

Department of Pathology, Wake Forest University Health

Sciences, Winston-Salem, NC, USA

\section{K. R. Geisinger}

Department of Internal Medicine (Gastroenterology), Wake Forest University Health Sciences, Winston-Salem, NC, USA

M. Davis · T. Nguyen · J. Sawyer · L. Rudel Department of Pathology-Lipid Sciences, Wake Forest University Health Sciences, Winston-Salem, NC, USA habitus may affect properties of the scan. We hypothesized that in high BMI patients nc-CT attenuation measurements expressed in Hounsfield units (HU) accurately estimate liver triglyceride concentrations as well as histological macrosteatosis.

Methods With informed consent, 15 patients underwent nc-CT scan of the abdomen prior to weight loss surgery with intraoperative wedge and core needle liver biopsy. Mean left lobe nc-CT Hounsfield units $\left(\mathrm{CT}_{\mathrm{L}}\right)$, liver TG (mg/g Pr), HMS (\%), BMI $\left(\mathrm{kg} / \mathrm{m}^{2}\right)$, liver-spleen index $\left(\mathrm{CT}_{\mathrm{L} / \mathrm{S}}=\right.$ hepatic $\mathrm{HU} /$ splenic $\left.\mathrm{HU}\right)$, and liver-spleen difference $\left(\mathrm{CT}_{\mathrm{L}-\mathrm{S}}=\right.$ hepatic $\mathrm{HU}-$ splenic $\left.\mathrm{HU}\right)$ were a priori outcomes.

Results In 15 patients (11 female) with a BMI of $44.4 \pm 1.1$ (mean $\pm \mathrm{SEM}), \mathrm{CT}_{\mathrm{L} / \mathrm{S}}, \mathrm{CT}_{\mathrm{L}-\mathrm{S}}$, and $\mathrm{CT}_{\mathrm{L}}$ measures were significantly associated with liver TG concentrations $(r=$ $-0.80, P<0.001 ; r=-0.80, P<0.001$; and $r=-0.71$, $P<0.01$, respectively; Table 1$)$. Macrosteatosis (\%) and liver triglyceride concentration were positively associated $(r=0.83 ; P<0.0001)$. BMI did not correlate strongly to liver triglyceride $(r=0.44, P=\mathrm{NS})$.

Conclusion Estimates of liver fat obtained by nc- CT scans (esp. $\mathrm{CT}_{\mathrm{L} / \mathrm{S}}, \mathrm{CT}_{\mathrm{L}-\mathrm{S}}$ ) correlate to chemical measurement of liver triglyceride concentrations, suggesting non-contrasted CT may be a suitable non-invasive "gold standard" for hepatic steatosis quantification in these patients.

Keywords Computed tomography - Non-alcoholic fatty liver disease - Macrosteatosis · Liver-spleen index · Obesity $\cdot$ Hepatic attenuation $\cdot$ Triglyceride
Abbreviations
TG Triglyceride
BMI Body mass index
NAFLD Non-alcoholic fatty liver disease 


$\begin{array}{ll}\text { nc-CT } & \text { Non-contrasted computed tomography } \\ \mathrm{HU} & \text { Hounsfield units } \\ \mathrm{CT}_{\mathrm{L} / \mathrm{S}} & \text { Left lobe liver-spleen Index } \\ \mathrm{CT}_{\mathrm{L}-\mathrm{S}} & \text { Left lobe liver-spleen attenuation difference } \\ \mathrm{CT}_{\mathrm{L}} & \begin{array}{l}\text { Left lobe computed tomography liver } \\ \text { attenuation }\end{array} \\ \mathrm{CT}_{\mathrm{Liu}} & \begin{array}{l}\text { Left lobe computed tomography liver } \\ \text { attenuation adjusted via the "Liu" equation }\end{array} \\ \mathrm{CTr} & \begin{array}{l}\text { Right lobe computed tomography liver } \\ \text { attenuation }\end{array} \\ \mathrm{CTr}_{\mathrm{L} / \mathrm{S}} & \text { Right lobe liver-spleen Index }\end{array}$

\section{Introduction}

Non-alcoholic fatty liver disease (NAFLD) is an emerging epidemic in the United States and other industrialized countries [1]. Abnormal fat deposition in the liver has a significant rate of transition to cirrhosis [2] and hepatocellular carcinoma [3] and worsens patient response to viral hepatitis therapy [4]. It is estimated that one-third of Americans-and $80 \%$ of patients undergoing weight-loss surgery - have fatty liver, and that the sequelae of NAFLD will eventually supplant hepatitis $C$ virus liver disease as the number one indication for liver transplant [5]. Unfortunately, fatty liver disease likely has even farther reaching human health implications. Simple hepatic macrosteatosis (HMS), defined as macrosteatosis regardless of inflammation, ballooning, or fibrosis, has been linked to insulin resistance, obesity, hyperlipidemia [6], and atherosclerotic heart disease in humans [6,7]. Currently, human clinical investigation of hepatic steatosis and its response to therapy is limited by dependence upon liver biopsy, an invasive and sometimes unpleasant procedure that carries a very small risk of serious complication [8].

Several studies have evaluated noninvasive methods for estimation of HMS, including ultrasound, CT, and MRI [9, 10]. Generally, magnetic resonance imaging techniques seem to be preferred, especially when it is desirable to accurately estimate lower levels of hepatic macrosteatosis and liver triglyceride, since proton NMR is generally believed to better assess the actual liver triglyceride concentration. However, heavily cited MRI studies have extrapolated triglyceride concentration from animal models [11] or a small sample of ex vivo liver MRI results collected in a single study [12], so this assumption remains incompletely tested.

Multiple studies have attempted to correlate computed tomography (CT) findings of non-obese subjects to the degree of HMS demonstrated histologically on hematoxylin and eosin (H\&E) stained liver sections with mixed results [13-15]. Groups have employed an array of attenuation indices, usually comparing liver Hounsfield units (HU) against that of a low adipose internal standard (e.g., the spleen, portal vein, left ventricle, etc.). A very recent report by Liu et al. [16] has proposed an equation to modify CT attenuation data with subject BMI to better correlate HU with HMS in patients with $>5 \%$ macrosteatosis. However, H\&E assisted liver biopsy interpretation of steatosis may only be semiquantitative and susceptible to interobserver variability [17] and sampling error [18]. Computer and Oil Red $\mathrm{O}$ stain have been proposed to improve precision, but have not been validated chemically either. As a result, histology as a "gold standard" may confound the evaluation of a CT scan's ability to accurately reflect liver fat.

Since no group has correlated CT findings with actual laboratory quantification of hepatic triglyceride per gram of liver protein, our aim is to discern whether or not unenhanced CT determination of hepatic macrosteatosis based on image attenuation data (Hounsfield units) is significantly correlated to chemical measurement of liver triglyceride (mg/g protein) in high BMI patients, a growing, unstudied demographic often unsuitable for MRI. Verified correlation of these radiologic and laboratory parameters may facilitate the development of a reliable and noninvasive standard measurement of liver steatosis for both clinical and research objectives.

\section{Methods}

This work was an IRB approved prospective, cross sectional pilot study. From 2009 to 2010, 15 patients scheduled to undergo elective weight loss surgery (laparoscopic gastric banding or Roux-en-Y gastric bypass surgery) at Wake Forest University Baptist Hospital, Winston-Salem, NC, USA agreed to a preoperative non-contrasted abdominal CT scan, as well as the intraoperative collection of a wedge liver biopsy, a core needle liver biopsy, and $10 \mathrm{ml}$ of whole blood. Previous experiments in obese patients indicated a small sample size provided sufficient variations in HMS to test non-contrasted CT's ability to discern HMS across a broad spectrum [19]. Patients were excluded for a history of chronic liver disease other than NAFLD (macrosteatosis in the setting of hepatic ballooning, inflammation or fibrosis), a history of malignancy other than non-melanomatous skin cancer, an INR greater than 1.8, the need for therapeutic anticoagulation after surgery, a history of chronic inflammatory diseases including but not limited to rheumatoid arthritis, psoriasis, systemic lupus erythematosis, sarcoidosis, inflammatory bowel disease, greater than or equal to $105 \mathrm{~g}$ of ethanol per week, greater than or equal to $45 \mathrm{~g}$ in a given day per week, and refusal to participate. 


\section{Liver Biopsy}

For lipid analysis, wedge biopsies from the lateral left hepatic lobe ranging from $250-500 \mathrm{mg}$ were rinsed with saline and snap frozen in liquid nitrogen in the operating room before subsequent storage at $-80^{\circ} \mathrm{F}$. At the time of analysis, all lipids from minced pieces of liver (50-100 mg) were extracted in 2:1 chloroform:methanol overnight [20]. All lipid quantification assays were performed on aliquots of the chloroform extract. Measurement of triglycerides was determined after the addition of Triton$\times 100$ to solubilize lipids; after removal of the chloroform by evaporation, lipids were suspended in water and quantified with enzymatic assays (triglyceride [TG] and cholesterol by Roche Diagnostics and unesterified cholesterol by Wako Chemicals USA) [21].

A single intraoperative core needle liver biopsy was taken from the lateral left lobe of each subject with an 18-gauge spring-loaded MC1820 Bard $^{\mathrm{TM}}$ biopsy device. These core needle samples were immediately placed in $10 \%$ buffered formalin and submitted for histologic evaluation of steatosis, necroinflammation, ballooning, and fibrosis by a single expert pathologist using standardized scoring methods [22, 23]. Hematoxylin and eosin and trichrome slide preparations were performed per center standards. A semiquantitative measure of HMS was made by point counting hepatocytes at $400 \times$ optical magnification. An overlying grid of $35 \mu \mathrm{m}^{2}$ boxes was used to assist counting. The hepatocytes of five, non-overlapping fields that did not include a portal tract were hand counted, yielding percentages of hepatocytes as macrosteatotic (lipid droplets large enough to displace the nucleus and/or $\geq 15 \mu \mathrm{m}$ ), microsteatotic (multiple lipid droplets all smaller than the nucleus), and normal (no evidence of lipid droplets) [24]. Degrees of HMS were graded according to convention established by Kleiner et al. [23]: minimal, $<5 \%$; grade 1, 5-33\%; grade 2, 33-66\%; and grade 3, $>66 \%$. In order to better clinically describe our cohort, the NASH activity score (NAS) ranging from $0-8$ was calculated on a standardized grading system: HMS (scale of $0-3)$, lobular inflammation (0-3), and hepatocellular ballooning (0-2), with higher scores indicating increasing severity of NASH. NAS scores $\geq 5$ have been validated as diagnostic of NASH. Separately, fibrosis was staged $0-4$ as described by Brunt et al. [22].

\section{Computed Tomography}

The non-contrasted computed tomography (Lightspeed 16; GE Medical Systems, Milwaukee, WI) was performed on a helical scanner set to scan and reconstruct as follows: $0.8 \mathrm{~s}$ rotation time, full 8 detector rows, 2.5 slice thickness,
0.875:1 beam pitch, 8.75 table speed, interval 2.5 , SFOV large, DFOV 35, $140 \mathrm{kV}$, amperage $350 \mathrm{~mA}$. Images were reviewed by a single, expert radiologist blinded to biopsy data who determined hepatic attenuation with Hounsfield units (HU) in hepatic regions corresponding to the subsequent left liver biopsy site. CT attenuation of three distinct circular areas was measured in the liver biopsy site (lateral left lobe) as well as the spleen to generate mean values. Care was taken to avoid inclusion of visually distinct vasculature and biliary structures in the regions of interest.

In addition to simple mean left lobe hepatic attenuation $\left(\mathrm{CT}_{\mathrm{L}}\right)$, four other indices derived from left lobe $\mathrm{CT}$ data were recorded for comparison to biopsy proven hepatic macrosteatosis and to chemical measures of hepatic triglyceride concentration. Splenic attenuation was used to calculate a liver-spleen Index $\left(\mathrm{CT}_{\mathrm{L} / \mathrm{S}}=\right.$ mean hepatic $\mathrm{HU} /$ mean splenic $\mathrm{HU}$ ) as well as liver-spleen attenuation difference $\left(\mathrm{CT}_{\mathrm{L}-\mathrm{S}}=\right.$ mean hepatic $\mathrm{HU}-$ mean splenic $\left.\mathrm{HU}\right)$. Also, liver attenuation data from the region of interest was transformed using the equation $\mathrm{CT}_{\mathrm{Liu}}=47.7+1.48 \mathrm{BMI}$ - 1.18CT Hounsfield units [16]. Liu et al. describe $\mathrm{CT}_{\text {Liu }}$ as correlating strongly to macrosteatosis in living liver donor candidates with HMS $>5 \%$. Mean CT attenuation from the non-biopsied, right lobe (CTr) was also averaged from three distinct areas and compared to the biopsied (lateral left) lobe's chemical, histological, and CT data to gauge the applicability of findings throughout the entire liver.

\section{Statistical Analysis}

Data were analyzed using descriptive statistical analysis, including mean with standard error for continuous variables and proportions for categorical variables. Comparisons between groups were done using chi-square or Fisher's exact tests for proportions and the Student's $t$ tests for continuous variables. Pearson product-moment correlation coefficients were calculated for continuous variables to determine strength of the correlation statistic. The significance of correlations were calculated from the t-statistic using the formula $t=\left(v r^{2} / 1-r^{2}\right)^{1 / 2}$ where $v=n-2$ and $r=$ correlation coefficient. Log transformations were used to normalize the distributions of data when needed.

\section{Results}

After informed consent, 15 patients (11 females) with a mean BMI of $44.4 \pm 1.1 \mathrm{~m} / \mathrm{kg}^{2}$ (range of 38.3-51.5) underwent liver biopsy (Table 1). Although the dataset is small, a wide variation in HMS was observed so that regression analysis can be appropriately used to compare 


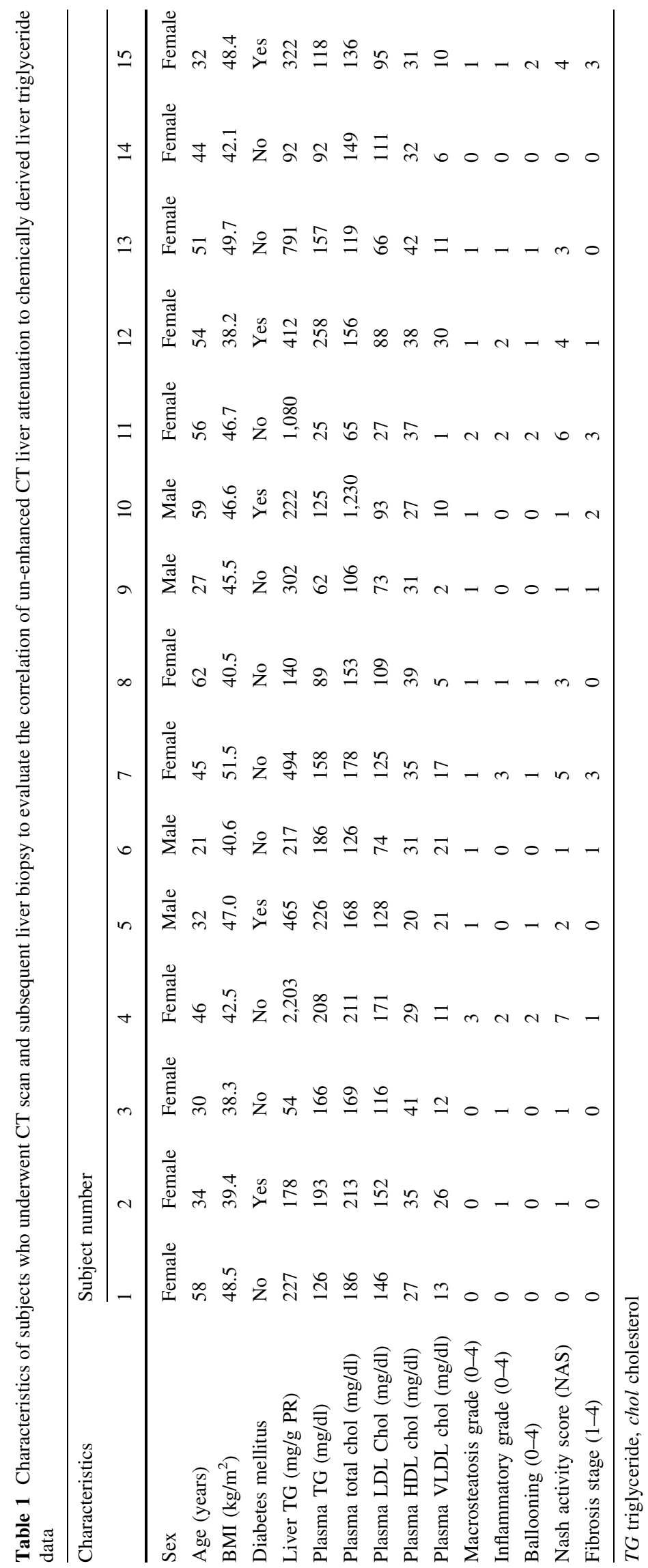


liver triglyceride concentration $(\mathrm{mg} / \mathrm{g}$ liver protein) after $\log$ transformation to both $\mathrm{CT}_{\mathrm{L} / \mathrm{S}}(r=-0.80, P<0.001$, $y=-0.6326 \times+2.5451)$ (Fig. 1$)$ and $\mathrm{CT}_{\mathrm{L}-\mathrm{S}}(r=-0.80$,

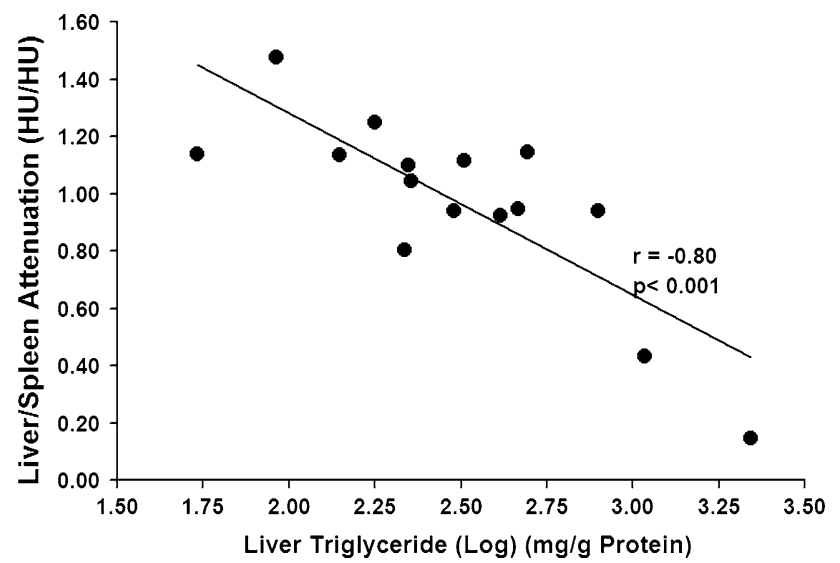

Fig. 1 Correlation of liver spleen index to liver triglyceride. Liver spleen index [liver attenuation (HU)/splenic attenuation (HU)] correlates strongly with chemical measurement of liver triglyceride $(\mathrm{mg} / \mathrm{gPR})(r=-0.80, P<0.001, n=15)$
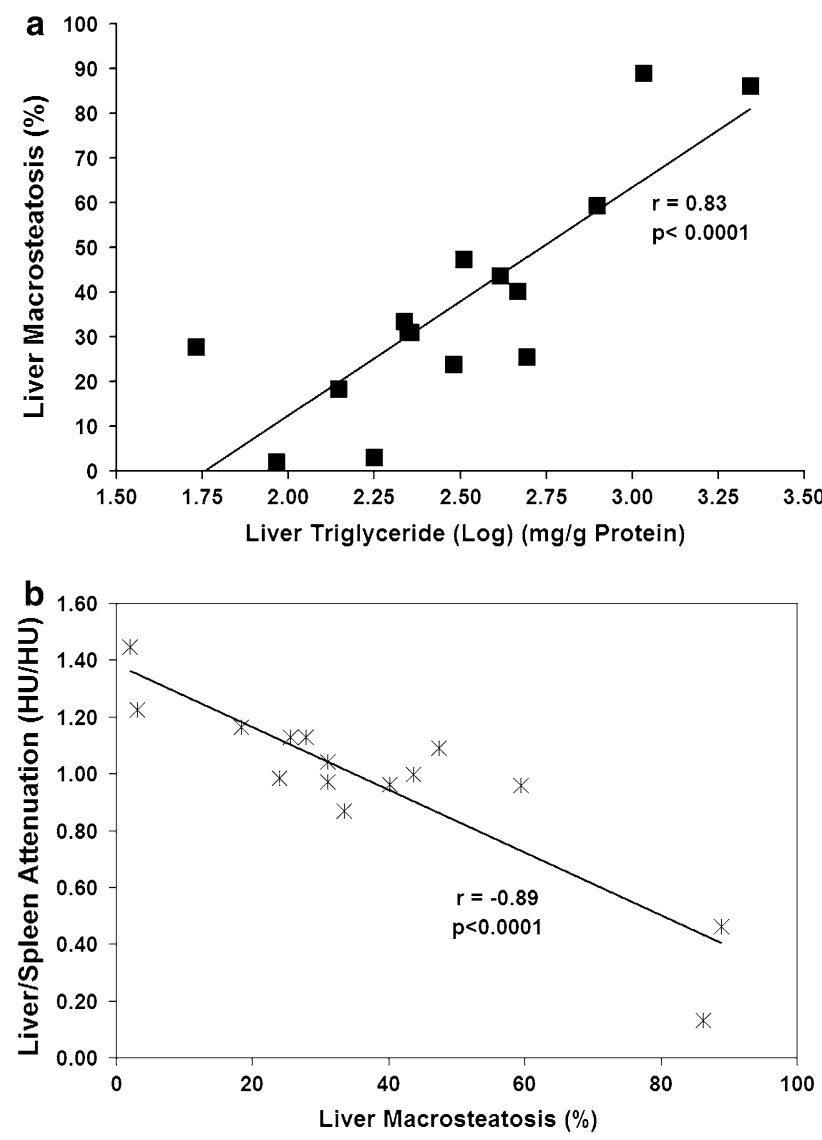

Fig. 2 Correlation of liver macrosteatosis (\%) to liver triglyceride (a) and liver spleen index $\left(\mathrm{CT}_{\mathrm{L} / \mathrm{S}}\right)(\mathbf{b})$. Histological macrosteatosis (HMS) correlates strongly with chemical measurement of liver triglyceride $(\mathrm{mg} / \mathrm{g}$ PR $)(r=0.83, P<0.0001, n=15)$ and $\mathrm{CT}_{\mathrm{L} / \mathrm{S}}$ $(r=-0.89, P<0.0001, n=15)$

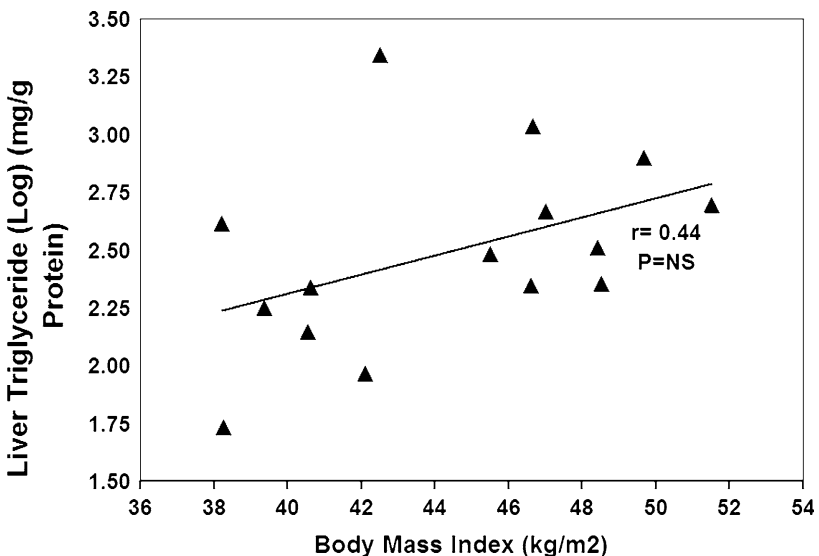

Fig. 3 Correlation of BMI to liver triglyceride. Subject BMI $\left(\mathrm{kg} / \mathrm{m}^{2}\right)$ does not correlate significantly with liver triglyceride $(\mathrm{mg} / \mathrm{g}$ protein) $(r=0.44, P>0.05, n=15)$

$P<0.001$ ) (data not shown). The correlations were strong but statistically significant. Histological macrosteatosis (\%) was similarly correlated to the chemical measurement of liver triglyceride $(r=0.83, P<0.0001)$ and $\mathrm{CT}_{\mathrm{L} / \mathrm{S}}(r=$ $-0.89, P<0.0001)$ (Fig. 2a, b). Neither $\mathrm{CT}_{\mathrm{L}}(r=-0.71$, $P<0.01)$ nor BMI-adjusted nc-CT data as proposed by Liu et al. $(r=0.80, P=0.001)$ reflected liver triglyceride (mg/g liver protein) any more closely than $\mathrm{CT}_{\mathrm{L} / \mathrm{S}}$.

Mean CT liver-spleen index from the right (non-biopsied) liver lobe $\left(\mathrm{CTr}_{\mathrm{L} / \mathrm{S}}\right)$ also correlated strongly to $\log$ transformed liver triglyceride $(r=-0.79, P<0.01)$ and HMS $(r=-0.88, P<0.001)$, while mean biopsied left liver lobe attenuation $\left(\mathrm{CT}_{\mathrm{L}}\right)$ and non-biopsied liver attenuation (CTr) was even more similar $(r=0.96, P<0.0001$, $n=15$ ). BMI was only marginally and nonsignificantly correlated with liver triglyceride concentration, the definitive measure of hepatic fat ( $r=0.44, P>0.05)$ (Fig. 3).

Seventy-three percent of subjects demonstrated at least mild HMS by surgical pathology evaluation $(n=11)$. Sixty percent and $53 \%$ of participants were found to have necroinflammation $(n=9)$ and/or at least stage 1 fibrosis $(n=11)$, respectively. Four biopsies revealed stage 3 fibrosis, and no subjects had cirrhosis. Mean percentage of histological macrosteatosis was $27 \pm 5.9 \%$. Three subjects met the predetermined criteria for steatohepatitis with a NASH activity score (NAS) $\geq 5$. Individual NAS ranged from 0-6. The liver triglyceride concentrations distributed widely from $54-2,203 \mathrm{mg} / \mathrm{g}$ of liver protein with a mean $( \pm$ sem $)$ value of $480 \pm 142 \mathrm{mg} / \mathrm{g} \operatorname{Pr}(n=15)$.

\section{Discussion}

This study has confirmed the utility of using nc-CT scanning to noninvasively evaluate the extent of liver 
triglyceride and hepatic macrosteatosis (HMS) in very high BMI patients, a growing population previously not studied with CT. The Hounsfield units obtained from the CT scan were significantly and directly correlated to the chemical assessment of hepatic triglyceride and to a standardized histologic estimation of HMS. While the number of patients examined in this study was small, the correlation extended across a quite wide range of liver triglyceride concentrations (Fig. 1) so that the measurements are reasonably robust and provide good estimates of triglyceride concentrations across the range of minimal to very high macrosteatosis. CT assessment of hepatic steatosis in earlier studies was criticized on the basis that the modality provided limited accuracy to estimate hepatic fat concentration accurately where this was low [15]. However, such criticism cannot be leveled at our dataset.

A strength of this study is that it is the first direct comparison of CT attenuation to chemical hepatic triglyceride concentrations of which we are aware. Multiple groups have reported that non-contrasted hepatic CT findings significantly correlate to HMS estimated by a single observer [14-16]. When reviewed together, these studies provide the impression that nc-CT scan may be of value in measuring liver fat above $30 \%$ HMS or when adjusted for individual BMI. However, all of these studies rely on the assumption that estimation of HMS from a core liver biopsy accurately represents in vivo liver triglyceride concentration. Until now this assumption has not been tested in humans.

Additionally, CT data of the non-biopsied right liver lobe (CTr) correlated strongly to liver TG, HMS, and CT attenuation of the biopsied lobe. In the context of previously published strong correlations in HMS between paired liver biopsies, such findings suggest our liver fat measures (HMS, TG, CT) apply broadly to most of the liver, thereby allaying a common concern in non-invasive hepatic diagnostic studies [18]. Lastly, this work chemically validates the use of a CT "Liver Spleen Index" (or ratio) to control for an individual subjects' scanning characteristics.

Our study has important limitations including small cohort size and the inclusion of only very high BMI subjects. However, in designing our study we believe that the existing data supporting nc-CT and our knowledge of the wide spectrum of HMS within a bariatric surgery cohort justified a small sample size both from a statistical and an ethical standpoint, not least given the potential risks associated with wedge liver biopsies and radiation exposure for non-clinical purposes. Also, we must acknowledge that a liver biopsy still remains essential for the determination of histological features of hepatocyte ballooning, inflammation and fibrosis that are of considerable importance in the full characterization of fatty liver disease. Finally, we are aware that the lifetime risk of medical radiation exposure is an emerging concern for clinicians [25]. The radiation exposure of the CT measures used here is relatively modest, however, and is confined to an abdominal region not involving the entire body, so the risk for such a procedure remains low.

In summary, we believe the novel observation that nc-CT hepatic attenuation closely reflects both hepatic macrosteatosis and chemically measured liver triglyceride across a wide range of steatosis $(54-2,203 \mathrm{mg} / \mathrm{g} \mathrm{Pr}$ ), eliminating interobserver variability, offers many interesting possibilities for the care of liver patients and liver donors. A convenient, validated measure of HMS alone would be a boon to clinicians and researchers, attempting to unravel the complex interplay between liver lipid burden and human metabolism as a whole. Based on our results, unenhanced computed tomography may represent liver triglyceride accurately enough to fill such a role. Confirmation of our results by a larger study would ultimately serve to elucidate the risk and benefits of this proposed use of computed tomography in obese and non-obese patients alike.

Open Access This article is distributed under the terms of the Creative Commons Attribution Noncommercial License which permits any noncommercial use, distribution, and reproduction in any medium, provided the original author(s) and source are credited.

\section{References}

1. Flegal KM, Carroll MD, Ogden CL, Johnson CL. Prevalence and trends in obesity among US adults, 1999-2000. JAMA. 2002;288:1723-1727.

2. Nair S, Mason A, Eason J, Loss G, Perrillo RP. Is obesity an independent risk factor for hepatocellular carcinoma in cirrhosis? Hepatology. 2002;36:150-155.

3. Wanless IR, Lentz JS. Fatty liver hepatitis (steatohepatitis) and obesity: An autopsy study with analysis of risk factors. Hepatology. 1990;12:1106-1110.

4. Adinolfi LE, Gambardella M, Andreana A, Tripodi MF, Utili R, Ruggiero G. Steatosis accelerates the progression of liver damage of chronic hepatitis $\mathrm{C}$ patients and correlates with specific HCV genotype and visceral obesity. Hepatology. 2001;33:1358-1364.

5. Browning JD, Szczepaniak LS, Dobbins R, et al. Prevalence of hepatic steatosis in an urban population in the United States: Impact of ethnicity. Hepatology. 2004;40:1387-1395.

6. Marchesini G, Bugianesi E, Forlani G, et al. Nonalcoholic fatty liver, steatohepatitis, and the metabolic syndrome. Hepatology. 2003;37:917-923.

7. Xirouchakis E, Sigalas A, Manousou P, et al. Models for nonalcoholic fatty liver disease: A link with vascular risk. Curr Pharm Des. 2008;14:378-384.

8. Rockey DC, Caldwell SH, Goodman ZD, Nelson RC, Smith AD. Liver biopsy. Hepatology. 2009;49:1017-1044.

9. Qayyum A, Chen DM, Breiman RS, et al. Evaluation of diffuse liver steatosis by ultrasound, computed tomography, and magnetic resonance imaging: Which modality is best? Clin Imaging. 2009;33:110-115

10. Schwenzer NF, Springer F, Schraml C, Stefan N, Machann J, Schick F. Non-invasive assessment and quantification of liver 
steatosis by ultrasound, computed tomography and magnetic resonance. J Hepatol. 2009;51:433-445.

11. Szczepaniak LS, Babcock EE, Schick F, et al. Measurement of intracellular triglyceride stores by $\mathrm{H}$ spectroscopy: Validation in vivo. Am J Physiol. 1999;276:E977-E989.

12. Vuppalanchi R, Cummings OW, Saxena R, et al. Relationship among histologic, radiologic, and biochemical assessments of hepatic steatosis: A study of human liver samples. J Clin Gastroenterol. 2007;41:206-210.

13. Lee SW, Park SH, Kim KW, et al. Unenhanced CT for assessment of macrovesicular hepatic steatosis in living liver donors: Comparison of visual grading with liver attenuation index. Radiology. 2007;244:479-485.

14. Limanond P, Raman SS, Lassman C, et al. Macrovesicular hepatic steatosis in living related liver donors: Correlation between CT and histologic findings. Radiology. 2004;230:276-280.

15. Park SH, Kim PN, Kim KW, et al. Macrovesicular hepatic steatosis in living liver donors: Use of CT for quantitative and qualitative assessment. Radiology. 2006;239:105-112.

16. Liu ZJ, Gong JP, Yan LN. Quantitative estimation of the degree of hepatic macrovesicular steatosis in a disease-free population: A single-center experience in mainland China. Liver Transpl. 2009;15:1605-1612.

17. El-Badry AM, Breitenstein S, Jochum W, et al. Assessment of hepatic steatosis by expert pathologists: The end of a gold standard. Ann Surg. 2009;250:691-697.
18. Merriman RB, Ferrell LD, Patti MG, et al. Correlation of paired liver biopsies in morbidly obese patients with suspected nonalcoholic fatty liver disease. Hepatology. 2006;44:874-880.

19. Shores N, Fernandez A, Geisinger K, et al. Hepatic acyl-coa: Cholesterol o-acyltransferase 2 activity predicts hepatic steatosis in obese humans. [Abstract] Hepatology. 2009;50:768A.

20. Folch J, Lees M, Sloane-Stanley GH. A simple method for the isolation, purification of total lipides from animal tissues. $J$ Biol Chem. 1957;224:12.

21. Carr TP, Andresen CJ, Rudel LL. Enzymatic determination of triglyceride, free cholesterol, and total cholesterol in tissue lipid extracts. Clin Biochem. 1993;26:39-42.

22. Brunt EM, Janney CG, Di Bisceglie AM, Neuschwander-Tetri BA, Bacon BR. Nonalcoholic steatohepatitis: A proposal for grading and staging the histological lesions. Am J Gastroenterol. 1999;94:2467-2474.

23. Kleiner DE, Brunt EM, Van Natta M, et al. Design and validation of a histological scoring system for nonalcoholic fatty liver disease. Hepatology. 2005;41:1313-1321.

24. Franzen LE, Ekstedt M, Kechagias S, Bodin L. Semiquantitative evaluation overestimates the degree of steatosis in liver biopsies: A comparison to stereological point counting. Mod Pathol. 2005;18:912-916.

25. Smith-Bindman R. Is computed tomography safe? N Engl J Med. 2010;363:1-4. 